\title{
ON NEWMAN'S THEOREM FOR FINITE-TO-ONE OPEN MAPPINGS ON MANIFOLDS
}

\author{
L. F. MCAULEY AND ERIC E. ROBINSON
}

\begin{abstract}
We prove the following important generalization of a famous theorem by Newman: If $(M, d)$ is a closed manifold, there is an $\varepsilon>0$ such that if $Y$ is a closed manifold and $f: M \rightarrow Y$ is a finite-to-one open surjective mapping which is not a homeomorphism, then there is at least one $y \in Y$ such that $\operatorname{diam} f^{-1}(y) \geqslant \varepsilon$.

A version of the above theorem was first proved by Černavskii using rather complicated covering arguments. Our proof by comparison is much simpler and uses modern homology theory.
\end{abstract}

1. Introduction. In 1931 M. H. A. Newman [N] essentially proved that if $M$ is a connected topological manifold, then there is an $\varepsilon>0$ such that if $G$ is a finite group acting nontrivially on $M$, then there is at least one orbit of diameter $\geqslant \varepsilon$. P. A. Smith [S] later generalized Newman's Theorem to cohomology manifolds with fixed coverings. In 1969 Dress [D] gave a comparatively simple and more accessible proof of Newman's result for $Z_{p}$-actions using modern homology theory instead of the complicated simplical constructions and covering arguments of Newman and Smith, respectively. Now, it is well known that the collection $H$ of orbits obtained from the action of a finite group $G$ on a manifold $M$ forms a continuous decomposition of $M$, where each element of the decomposition is a finite set. In $1964 \mathrm{~A}$. V. Černavskii [C] generalized Newman's Theorem by showing that if $M$ is a topological manifold, and $U$ is a bounded open subset of $M$, then there is an $\varepsilon>0$ such that if $H$ is a continuous decomposition of $M$ into finite sets, at least one of the nondegenerate elements in $H$ which is contained in $U$ has diameter $\geqslant \varepsilon$. This is a true generalization of Newman's Theorem, for not all continuous decompositions can be formed by the actions of finite groups (see Robinson [R]).

There are many important results in the above paper by Cernavskii, but, again the proofs involve complicated covering arguments. Several of these results were simplified and modernized by Väisälä [V] for maps between closed manifolds. We wish here similarly to provide a relatively simple proof of Cernavskii's generalization of Newman's Theorem for closed manifolds and extend some of the results of Dress [D].

Received by the editors August 14, 1981 and, in revised form, June 28, 1982. Presented to the Society at the 789th meeting on November 17, 1981 in Amherst. Massachusetts.

1980 Mathematics Subject Classification. Primary 54C 10: Secondary 55M25, 55M35, 57S12, 57S17.

Key words and phrases. Manifold, continuous decomposition, proper open map, degree, group action. 
2. Terminology and preliminary results. It is well known that if $G$ is a continuous decomposition of a compact metric space $X$, where each element $g \in G$ consists of a finite set of points, then the projection map $\pi: X \rightarrow X / G$ onto the decomposition space is a finite-to-one proper (inverse image of a compact set is compact) open (image of an open set is open) mapping. Conversely, if $X$ and $Y$ are locally compact metric spaces and $f: X \rightarrow Y$ is a finite-to-one proper open surjective mapping, then $G=\left\{f^{-1}(y) \mid y \in Y\right\}$ forms a continuous decomposition of $X$ into finite sets. Thus, our generalization of Newman's Theorem is

THEOREM 3. If $(M, d)$ is a closed n-dimensional manifold, there is an $\varepsilon>0$ such that if $Y$ is a closed manifold and $f: M \rightarrow Y$ is a finite-to-one (proper) open surjective mapping which is not a homeomorphism, then there is at least one $y \in Y$ such that $\operatorname{diam} f^{-1}(y) \geqslant \varepsilon$.

If $f: M \rightarrow Y$ is a mapping, we let $B_{f}=\{x \in M \mid f$ is not a local homeomorphism at $x\}$. The set $B_{f}$ is called the branch set of $f$. The multiplicity of $x \in M$ is the cardinality of $f^{-1}(f(x))$.

We will need the following result found in $[\mathbf{C}, \mathbf{V}]$ :

THEOREM 1. If $f: M \rightarrow Y$ is a finite-to-one proper open surjective mapping where $M$ is an n-dimensional manifold, then $M-f^{-1}\left(f\left(B_{f}\right)\right)$ is the set of points of maximum multiplicity and is a dense open subset of $M$.

Now, let $f: M \rightarrow Y$ be a proper finite-to-one open mapping where $M$ is an $n$-dimensional connected manifold and $Y$ is a locally compact topological space. For each $x \in M^{n}$, a winding number, $w(x)$, of $f$ at $x$ is defined. If $x \in M^{n}-B_{f}$, then set $w(x)=1$. If $x \in B_{f}$, then set $w(x)$ equal to the integer $s$ specified as follows: Let $\beta=\min \left\{d\left(x_{1}, x_{2}\right) \mid x_{1}, x_{2} \in f^{-1}(f(x)), x_{1} \neq x_{2}\right\}$ or $\beta=1$ in case $\{x\}=f^{-1}(f(x))$. Using Theorem 4.6 in [W] and the fact that $f$ is proper, we may find an open set $R \subseteq Y$ such that $f(x) \in R, \bar{R}$ is compact, and each component of $f^{-1}(R)$ has diameter $<\beta / 2$. Let $\left\langle z_{i}\right\rangle$ be a sequence of points in $M-f^{-1}\left(f\left(B_{f}\right)\right)$ which converges to $x$. Since $f$ is proper and open, $\left\langle f^{-1}\left(f\left(z_{i}\right)\right)\right\rangle \rightarrow f^{-1}(f(x))$. Let $W$ be the component of $f^{-1}(R)$ containing $x$ and $s$ be the cardinality of $f^{-1}\left(f\left(z_{i}\right)\right) \cap W$, when $f^{-1}\left(z_{i}\right) \cap W \neq \varnothing$. Note that $f \mid W$ is a finite-to-one proper open mapping and that if $y \in f^{-1}\left(f\left(z_{i}\right)\right) \cap W$, then $f \mid W$ is a local homeomorphism at $y$. Consequently, using Theorem $1, f \mid W$ has maximum multiplicity at $y$. It follows that the cardinality of $f^{-1}\left(f\left(z_{i}\right)\right) \cap W$ is the same for all $i$ such that $f^{-1}\left(f\left(z_{i}\right)\right) \cap W \neq \varnothing$. This shows that $s$ is independent of the particular sequence $\left\langle z_{i}\right\rangle$ chosen in $\bar{U}-f^{-1}\left(f\left(B_{f}\right)\right)$, which converges to $x$. To see that $s$ is also independent of the choice of $R$ and $w$, let $R^{\prime}$ be any other open set in $Y$ such that $f(x) \in R^{\prime}, \bar{R}^{\prime}$ is compact, and each component of $f^{-1}\left(R^{\prime}\right)$ has diameter $<\beta / 2$. Let $W^{\prime}$ be the component of $f^{-1}\left(R^{\prime}\right)$ which contains $x$. We may assume without any loss that $\bar{R}^{\prime} \subseteq R$. It follows that $\bar{W}^{\prime} \subseteq W$. Clearly, for each $i$, the cardinality of $f^{-1}\left(f\left(z_{i}\right)\right) \cap W^{\prime}$ is less than or equal to $s$. It suffices to show that this cardinality is not less than $s$, whenever $f^{-1}\left(f\left(z_{i}\right)\right) \cap W^{\prime} \neq \varnothing$. So, suppose there is a $j$ such that the cardinality of $f^{-1}\left(f\left(z_{j}\right)\right) \cap W^{\prime}$ is $t$ where $1 \leqslant t \leqslant r$. Again, from Theorem 1 it follows that the cardinality of $f^{-1}\left(f\left(z_{i}\right)\right) \cap W^{\prime}$ is $t$, 
whenever $f^{-1}\left(f\left(z_{i}\right)\right) \cap W^{\prime} \neq \varnothing$. Since $\left\langle f^{-1}\left(f\left(z_{i}\right)\right)\right\rangle \rightarrow f^{-1}(f(x))$ and $x \in W^{\prime}$, we can choose $I$ such that $f^{-1}\left(f\left(z_{i}\right)\right) \cap W^{\prime} \neq \varnothing$, whenever $i \geqslant I$. Since $t<s$, we may choose $y_{i} \in f^{-1}\left(f\left(z_{i}\right)\right) \cap\left(W-W^{\prime}\right)$ for $i \geqslant I$. Now $\bar{W}$ is compact since $f$ is proper, and, hence, $\left\{y_{i} \mid i \geqslant I\right\}$ has at least one limit point $y \in \bar{W}$. Then, $y \in f^{-1}(f(x)) \cap$ $\left(W-W^{\prime}\right)$. But, $f^{-1}(f(x)) \cap \bar{W}=\{x\}$. Hence, $y=x$, which is impossible since $x \in W^{\prime}$.

Next, suppose $h: M^{n} \rightarrow \mathbf{R}^{k}$ is an imbedding and $x^{*} \in M^{n}-\left(f^{-1}\left(f\left(B_{f}\right)\right) \cup \partial M^{n}\right)$. Since $\left.f\right|_{M^{n}-f^{\prime}\left(f\left(B_{f}\right)\right)}$ is a covering map, we may choose an open set $V \subseteq Y$ such that $f\left(x^{*}\right) \in V$, and $f^{-1}(V)=\cup_{i=1}^{m} V_{i}$, where $\bar{V}_{i} \cap \bar{V}_{j}=\varnothing$ if $i \neq j, V_{i}$ is open, $\left.f\right|_{V_{1}}$ is a homeomorphism for each $i=1,2, \ldots, m$ and

$$
\overline{\bigcup_{i=1}^{m} V_{i}} \cap\left[f^{-1}\left(f\left(B_{f}\right)\right) \cup \partial M^{n}\right]=\varnothing .
$$

Without any loss we may assume that $x^{*} \in V_{1}$. Using the normality of $M^{n}$, define $k: M^{n} \rightarrow[0,1]$ such that $k(x)=1$, for $x \in M^{n}-\left[\cup_{i=2}^{m} V_{i}\right]$; and $k(x)=0$ for $x \in f^{-1}\left(f\left(x^{*}\right)\right) \cap V_{i}, i=2,3, \ldots, m$. Now define $g: M^{n} \rightarrow \mathbf{R}^{k}$ by

$$
g(x)=\sum_{z \in f^{-1}(f(x))} \frac{1}{m} w(z) k(z) h(z)
$$

(using the vector addition and scalar multiplication in $\mathbf{R}^{k}$ ). Since $f$ is proper and open and $Y$ is locally compact, $g$ is continuous and proper. Furthermore, if we define $j: Y \rightarrow g\left(M^{n}\right)$ by $j(y)=g(x)$ when $x \in j^{-1}(y)$, then $j$ is well defined, continuous, and the following diagram commutes:

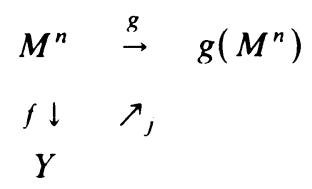

We will use the notion of degree of a mapping given by Dress in [D]. Namely, let $R$ be a commutative ring with unity and $f: M^{n} \rightarrow X$ a proper map, where $M^{n}$ is an $R$-oriented $n$-dimensional manifold and $X$ is a topological space. Then, for each compact subset $K$ of $M^{n}$, the degree of $f$ at $K: d(f, k)$, is $f_{*}\left(\theta_{K}\right)$, where $\theta_{k}$ is a fundamental class in $H_{n}\left(M^{n} ; M^{n}-f^{-1}(K) ; R\right)$ and $f_{*}: H_{n}\left(M^{n} ; M^{n}-f^{-1}(K) ; R\right)$ $\rightarrow H_{n}(X, X-K: R)$ is the map on homology induced by $f$. We will say that $f$ is essential at $K$ if $d(f, K) \neq 0$. Otherwise, we will say that $f$ is inessential at $K$. If $K=\{x\}$, we will say that $f$ is essential or inessential at $x$.

The following statements appearing in [D] are included for easy reference:

(1) If $K=f^{-1}\left(K^{\prime}\right) \subset K_{1}$ for some compact $K_{1}$ with $K_{1} \cap \partial M^{n}=\varnothing$, then $d\left(f, K^{\prime}\right)=f_{*}\left(\theta_{K_{1}}\right)$.

(2) $d\left(f, K^{\prime}\right)=d\left(g, K^{\prime}\right)$, if there is a homotopy $H: M^{n} \times I \rightarrow X$ such that $H(\cdot, 0)$ $=f, H(\cdot, 1)=g, H^{-1}\left(K^{\prime}\right)$ is compact and $H^{-1}\left(K^{\prime}\right) \cap\left(\partial M^{n} \times I\right)=\varnothing$.

(3) If $U \subseteq M^{n}$ is some open subset containing $K=f^{-1}\left(K^{\prime}\right)$, then $d\left(f, K^{\prime}\right)=$ $d\left(f \mid U, K^{\prime}\right)$.

(4) If $M^{n}=M_{1} \cup \dot{U} M_{2}$ (disjoint union), $f_{i}=f \mid M_{i}(i=1,2)$, then $d\left(f, K^{\prime}\right)=$ $d\left(f_{1}, K^{\prime}\right)+d\left(f_{2}, K^{\prime}\right)$. 
(5) If $f: M^{n} \rightarrow X$ is an open imbedding into an $R$-oriented, $n$-dimensional manifold and $K^{\prime} \subseteq f\left(M^{n}-\partial M^{n}\right)$, then $d\left(f, K^{\prime}\right)=\theta_{K}$.

(6) If $K^{\prime \prime} \subset K^{\prime}$, then $d\left(f, K^{\prime \prime}\right)$ is defined and is the restriction of $d\left(f, K^{\prime}\right) \in$ $H_{n}\left(X, X-K^{\prime} ; R\right)$ to $H_{n}\left(X, X-K^{\prime \prime} ; R\right)$. If especially $X$ is an $n$-dimensional, $R$-oriented manifold and $U \subseteq X$ open, such that $f^{-1}(U) \subseteq M^{n}-\partial M^{n}$ and relative compact in $M^{n}$, then $d(f, x) \in R$ is defined and locally constant for $x \in U$. If furthermore $M^{n}$ is compact, then $\operatorname{deg}(f, x)$ is defined and locally constant for all $x \in X-f\left(\partial M^{n}\right)$. So, if $\partial M^{n}=\varnothing$ and $X$ is connected, $d(f, x)$ is independent of $x \in X$ and just the usual degree of $f$.

(7) If $g: X \rightarrow Y$ is another map and $K^{\prime}=g^{-1}\left(K^{\prime \prime}\right)$ for some $K^{\prime \prime} \subset Y$, then $d\left(g \circ f, K^{\prime \prime}\right)=g_{*} d\left(f, K^{\prime \prime}\right)$, especially $g \circ f$ is inessential at $K^{\prime \prime}$, if $f$ is inessential at $K^{\prime}$.

Now, let $f: M^{n} \rightarrow Y$ be a proper finite-to-one open mapping where each of $M^{n}$ and $Y$ are connected $n$-dimensional orientable topological manifolds. Let $m$ be the maximum multiplicity of $f, R=Z / m Z$ and $y \in Y-\partial Y$ such that $f^{-1}(y) \cap \partial M^{n}=$ $\varnothing$. We claim $f$ is inessential at $y$. Note that if $f^{-1}(y) \subseteq M^{n}-f^{-1}\left(f\left(B_{f}\right)\right)$, this follows from Theorem 1 and (3)-(5) since $\left.f\right|_{M^{n}-f^{\prime}\left(f\left(B_{f}\right)\right)}$ is a covering map. (If $f$ is orientation reversing at any point of $f^{-1}(y)$, then in fact, $d(f, y)=0$ for $R=Z$, and, hence, for $R=Z / m Z$.) If $y \in f\left(B_{f}\right)$, choose an open $n$-ball $B$ in $Y$ such that $f^{-1}(B) \cap \partial M^{n}=\varnothing$. By Theorem 1, choose $y_{0} \in B-f\left(B_{f}\right)$ and let $K: Y \times I \rightarrow Y$ be an isotopy such that $K:(x, t)=x$ for $x \in Y-B$ and $t \in I, K(b, 0)=b$, for all $b \in B$ and $K\left(y_{0}, 1\right)=y$. Now, define $H: M^{n} \times I \rightarrow Y$ by $H(x, t)=K(f(x), t)$. So, $H(x, 0)=f(x)$, for all $x \in M^{n}$. Let $g(x)=H(x, 1)$, for $x \in M^{n}$. Then, $g$ is a proper finite-to-one open mapping and $g^{-1}(y) \subseteq M^{n}-\left(g^{-1}(g(B g)) \cup \partial M^{n}\right)$. From the preceding discussion it follows that $d(g, y)=0$. It follows from (2) that $d(f, y)=$ $d(g, y)$. Hence, $f$ is inessential at $y$ as was claimed. It is interesting to note that if $f$ is orientation preserving, the local degree of $f$ at a point in $Y-\partial Y$ corresponds to the winding number of $f$ at its inverse if by local we mean $\left.f\right|_{w}$ where $W$ is the component used to define the winding number.

The following conclusion, which is analogous to Newman's Lemma in [D], will be useful:

LEMMA 1. Consider the commutative diagram

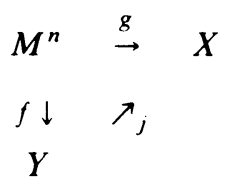

where $M^{n}$ and $Y$ are $n$-dimensional orientable manifolds, $X$ is a locally compact metric space, $f$ is a finite-to-one proper open surjective mapping, and $g$ and $j$ are proper mappings. Let $x \in X$ be such that $g^{-1}(x) \cap\left(\partial M^{n} \cup f^{-1}(\partial Y)\right)=\varnothing$ and $y \in j^{-1}(x)$. If $m$ is the maximum multiplicity of $f$, then $g$ is inessential at $y$ for $R=Z / m Z$.

Proof. It follows from the discussion above that $f$ is inessential at $y$ for each $y \in j^{-1}(x)$. Thus, since $j^{-1}(x)$ is compact, we see that $f$ is inessential at $j^{-1}(x)$. From (7) and the commutative diagram it follows that $g$ is inessential at $x$. 
3. Results. Newman's Theorem follows easily from the following extension of Lemma 3 in [D]:

THEOREM 2. Let $U$ be an open connected, relatively compact subset of $\mathbf{R}^{n}, f: \bar{U} \rightarrow Y$ a proper finite-to-one open mapping which is not a homeomorphism where $Y$ is an n-dimensional manifold,

$$
f^{-1}(\partial Y) \subseteq(\bar{U}-U), \quad D=\operatorname{Max}\{d(x, \bar{U}-U) \mid x \in U\},
$$

and

$$
C=\operatorname{Max}\left\{\operatorname{diam}\left(f^{-1}(f(x))\right) \mid x \in(\bar{U}-U)\right\}
$$

Then $D \leqslant C$.

Proof. Suppose $C<D$. Then there is an $x^{*} \in U$ with $d\left(x^{*}, \bar{U}-U\right)>C$. Using Theorem 1 and the fact that $f$ is open we may assume that $x^{*} \notin\left[f^{-1}\left(f\left(B_{f}\right)\right) \cup\right.$ $(\bar{U}-U)]$. Define $g: \bar{U} \rightarrow \mathbf{R}^{n}$ and $j: Y \rightarrow g(\bar{U})$ as in the preceding section with $h: \bar{U} \rightarrow \mathbf{R}^{n}$ the identity. That is,

$$
g(x)=\sum_{z \in f^{-1}(f(x))} \frac{1}{m} w(z) k(z) z,
$$

where $m$ is the maximum multiplicity of $f$. Note $g\left(x^{*}\right)=x^{*}$. We claim that $g^{-1}\left(x^{*}\right) \cap(\bar{U}-U)=\varnothing$. For suppose $z \in g^{-1}\left(x^{*}\right) \cap(\bar{U}-U)$. Then

$$
\begin{aligned}
C & <d\left(x^{*}, \bar{U}-U\right) \leqslant\left\|x^{*}-z\right\|=\|g(z)-z\| \\
& \leqslant \sum_{x \in f^{-1}(f(z))} \frac{1}{m} w(x) k(x)\|x-z\| \\
& \leqslant \sum_{x \in f^{-1}(f(z))} \frac{1}{m} w(x) k(x) \operatorname{diam} f^{-1}(f(z)) \\
& =\operatorname{diam} f^{-1}(f(z))=C,
\end{aligned}
$$

which is a contradiction. It follows from Lemma 1 that $g$ is inessential at $x^{*}$ for $R=Z / m Z$. Now, define the proper homotopy $H: \bar{U} \times I \rightarrow R^{n}$ by $H(x, t)=t x+$ $(1-t) g(x)$. We claim $H\left(x^{*} \times I\right) \cap H(\bar{U}-U \times I)=\varnothing$. For if not, there is a $z \in \bar{U}-U$ and $s, t \in I$ such that $H\left(x^{*}, s\right)=H(z, t)$. Thus, $x^{*}=t z+(1-t) z$. However, then

$$
\begin{aligned}
C & <d\left(x^{*}, \bar{U}-U\right) \leqslant\left\|x^{*}-z\right\|=\left\|x^{*}-t z-(1-t) z\right\| \\
& =(1-t)\|g(z)-z\| \leqslant(1-t) \sum_{x \in f^{-1}(f(z))} \frac{1}{m} w(x) k(x)\|x-z\| \\
& \leqslant(1-t) \sum_{x \in f^{-1}(f(z))} \frac{1}{m} w(x) k(x) \operatorname{diam} f^{-1}(f(z)) \\
& =(1-t) \operatorname{diam} f^{-1}(f(z)) \leqslant C,
\end{aligned}
$$

which is also a contradiction. Now, from (2) we get that $0=d\left(g, x_{*}\right)=$ $g\left(H_{0}, H_{0}\left(x_{*}\right)\right)=d\left(H_{1}, H_{2}\left(x^{*}\right)\right)$. But, $H_{1}$ is the identity and so $d\left(H_{1}, H_{1}\left(x^{*}\right)\right) \neq 0$, a contradiction. Hence $C \Varangle D$ and the theorem follows. 
We will now give a proof of Theorem 3 that closely parallels Newman's original argument.

Proof of Theorem 3. Let $D_{i}$ be the standard open $n$-dimensional ball of radius $i$ in $R^{n}$. For each $x \in M$, choose a chart $\phi_{x}: D_{3} \rightarrow M$. Using the compactness of $M$ we can cover $M$ with a finite number of sets $\phi_{1}\left(D_{3}\right), \phi_{2}\left(D_{3}\right), \ldots, \phi_{k}\left(D_{3}\right)$. Furthermore, there is an $\varepsilon>0$ such that if $x \in M, x \in \phi_{i}\left(D_{3}\right)$ for some $i=1,2, \ldots, k$ and $y \in D_{2}$ such that $d\left(x, \phi_{i}(y)\right)<\varepsilon$, then there is a $y^{1} \in D_{3}$ such that $\phi\left(y^{1}\right)=x$ and $\left\|y-y^{1}\right\|$ $\leqslant \frac{1}{2}$. Now suppose $f: M^{n} \rightarrow Y$ is a finite-to-one (proper) open surjective mapping where $Y$ is a closed manifold such that $\operatorname{diam}\left(f^{-1}(f(x))\right)<\varepsilon$, for each $x \in M$, and there is an $x^{*} \in M$ such that $f^{-1}\left(f\left(x^{*}\right)\right)$ is nondegenerate. Without any loss we may assume that $x^{*}=\phi_{1}(0)$, where 0 is the origin in $\mathbf{R}^{n}$. Let $V$ be the component of $f^{-1}\left(f\left(\phi_{1}\left(D_{1}\right)\right)\right)$ which contains $x^{*}$. Then $f(\bar{V})=\overline{f\left(\phi_{1}\left(D_{1}\right)\right)}, \bar{V} \cap\left[f^{-1}\left(\overline{f\left(\phi_{1}\left(D_{1}\right)\right)}-\right.\right.$ $\left.\left.f\left(\phi_{1}\left(D_{1}\right)\right)\right)\right] \subseteq \bar{V}-V,\left.f\right|_{V}$ is a proper, finite-to-one open mapping onto $f\left(\phi_{1}\left(D_{1}\right)\right)$, and $\bar{V} \subseteq \phi_{1}\left(D_{2}\right)$. Let $\bar{U}=\phi_{1}^{-1}(\bar{V})$ and $f^{\prime}=\left.\left.f\right|_{\bar{V}} \circ \phi_{1}\right|_{\bar{U}}$. Then $f^{\prime}$ is a finite-toone proper open mapping which is not a homeomorphism satisfying the hypotheses of Theorem 2. However, $\operatorname{Max}\left\{\operatorname{diam} f^{\prime-1}\left(f^{\prime}(x)\right) \mid x \in \bar{U}-U\right\}<1 \leqslant$ $\operatorname{Max}\{d(x, \bar{U}-U)\}$. This contradicts Theorem 2. Hence, Theorem 3 follows.

\section{REFERENCES}

[C] A. V. Cernavskii, Finite-to-one open mappings of manifolds, Mat. Sb. (1964), 357-369.

[D] A. Dress, Newman's theorem on transformation groups. Topology 8 (1969), 203-207.

[M] Deane Montgomery, Remark on continuous collections (to appear).

[N] M. H. A. Newman, A theorem on periodic transformations of spaces, Quart. J. Math. Oxford Ser. 2 (1931), 1-9

[R] E. E. Robinson, A characterization of certain branched coverings as group actions, Fund. Math. 103 (1979), 43-45.

[S] P. A. Smith. Transformations of finite period. III. Newman's Theorem, Ann. of Math. (2) 42 (1941). 446-458.

[V] J. Väisälä, Discrete open mappings on manifolds, Ann. Acad. Sci. Fenn. Ser. A I Math. (1966).

[W] G. T. Whyburn, Analitic topologi, Amer. Math. Soc. Colloq. Publ., vol. 28. Amer. Math. Soc., Providence, R. I., 1942.

Department of Mathematical Sciences, State University of New York at Binghamton, BINGHAMTON, NEW YORK 13901

Department of Mathematics, Ithaca College, Ithaca, New York 14850 